\title{
Antinociceptive role of neurotensin receptor 1 in rats with chemotherapy-induced peripheral neuropathy
}

\author{
Mei Yin ${ }^{1,2}$, Yeo-Ok Kim ${ }^{1}$, Jeong-ll Choi ${ }^{1}$, Seongtae Jeong ${ }^{1}$, Si-Ho Yang ${ }^{1}$, Hong-Beom Bae ${ }^{1,2}$, and \\ Myung-Ha Yoon ${ }^{1,2}$
}

'Department of Anesthesiology and Pain Medicine, Chonnam National University Medical School, Gwangju, Korea

${ }^{2}$ The Brain Korea 21 Project, Center for Biomedical Human Resources at Chonnam National University, Gwangju, Korea

Received May 11, 2020

Revised July 21, 2020

Accepted July 21, 2020

Handling Editor: Jong Yeon Park

\section{Correspondence}

Hong-Beom Bae

Department of Anesthesiology and Pain Medicine, Chonnam National University Medical School, 160, Baekseo-ro, Donggu, Gwangju 61469, Korea

Tel: +82-62-220-6895

Fax: +82-62-232-6294

E-mail: nextphil2@jnu.ac.kr

Myung-Ha Yoon

Department of Anesthesiology and Pain Medicine, Chonnam National University Medical School, 160, Baekseo-ro, Donggu, Gwangju 61469, Korea

Tel: +82-62-220-6895

Fax: +82-62-232-6294

E-mail: mhyoon@jnu.ac.kr
Background: Chemotherapy-induced peripheral neuropathy (CIPN) is a major side effect of anti-cancer drugs. Neurotensin receptors (NTSRs) are widely distributed within the pain circuits in the central nervous system. The purpose of this study was to determine the role of NTSR1 by examining the effects of an NTSR1 agonist in rats with CIPN and investigate the contribution of spinal serotonin receptors to the antinociceptive effect.

Methods: Sprague-Dawley rats (weight 150-180 g) were used in this study. CIPN was induced by injecting cisplatin $(2 \mathrm{mg} / \mathrm{kg}$ ) once a day for 4 days. Intrathecal catheters were placed into the subarachnoid space of the CIPN rats. The antiallodynic effects of intrathecally or intraperitoneally administered PD 149163, an NTSR1 agonist, were evaluated. Furthermore, the levels of serotonin in the spinal cord were measured by high-performance liquid chromatography.

Results: Intrathecal or intraperitoneal PD 149163 increased the paw withdrawal threshold in CIPN rats. Intrathecal administration of the NTSR1 antagonist SR 48692 suppressed the antinociceptive effect of PD 149163 given via the intrathecal route, but not the antinociceptive effect of intraperitoneally administered PD 149163. Intrathecal administration of dihydroergocristine, a serotonin receptor antagonist, suppressed the antinociceptive effect of intrathecally administered, but not intraperitoneally administered, PD 149163. Injecting cisplatin diminished the serotonin level in the spinal cord, but intrathecal or intraperitoneal administration of PD 149163 did not affect this reduction.

Conclusions: NTSR1 played a critical role in modulating CIPN-related pain. Therefore, NTSR1 agonists may be useful therapeutic agents to treat CIPN. In addition, spinal serotonin receptors may be indirectly involved in the effect of NTSR1 agonist.

Key Words: Cisplatin; Dihydroergocristine; Hyperalgesia; Neurotensin; Neuralgia; PD 149163; Peripheral Nervous System Diseases; Serotonin Antagonists; Spinal Cord; SR 48692.

\section{INTRODUCTION}

Chemotherapy-induced peripheral neuropathy (CIPN) is one of the most severe and long-lasting side effects caused by chemotherapeutic agents [1]. Symptoms of CIPN, such as abnormal touch sensation, altered responses to thermal (c) This is an open-access article distributed under the terms of the Creative Commons Attribution Non-Commercial License (http://creativecommons.org/licenses/by-nc/4.0/), which permits unrestricted non-commercial use, distribution, and reproduction in any medium, provided the original work is properly cited.

(c) The Korean Pain Society, 2020
Author contributions: Mei Yin: Writing/manuscript preparation; Yeo-OK Kim: Investigation; Jeong-II Choi: Study conception; Seongtae Jeong: Study conception; Si-Ho Yang: Formal analysis; Hong-Beom Bae: Writing/manuscript preparation; Myung-Ha Yoon: Supervision. 
stimuli, and pain, which are often dose dependent and progressively worse during and after chemotherapy [2], typically develop in the "glove and stocking" areas of the distal extremities [3]. Although medications have been employed to treat the symptoms associated with CIPN, few medications have been consistently effective or tolerable [4-6]. Also, despite intense efforts, no definite analgesics have been developed to treat CIPN.

Neurotensin (NTS) is an endogenous 13-amino acid peptide, originally isolated from bovine hypothalamus [7]. This neuropeptide and its receptors are widely distributed in the central nervous system (CNS), including sites important for pain transmission and modulation [8]. The multiple effects of NTS derive from its interaction with three different receptors (NTSR1, NSTR2, NSTR3). Microinjection of NTS, or NTS analogs, to specific brain areas exerts various biological effects through interactions with neurons secreting dopamine, serotonin, or norepinephrine [9-11]. Furthermore, NTS1 agonists have shown antinociceptive effects in various pain models. For example, intrathecal injection of NTSR1 agonist dose-dependently attenuates formalin-induced pain behavior in rats [12], and systemic administration of NTSR1 analogs mediates the visceral analgesic effects of NTS in the writhing model [13]. Moreover, intrathecal delivery of NTSR1 agonists reversed heat hyperalgesia and tactile allodynia induced by chronic neuropathic pain models, such as sciatic nerve constriction [14].

Serotonin (5-hydroxytryptamine, 5-HT) is involved in either facilitatory or inhibitory nociceptive transmission via the activation of different 5-HT subtype receptors [15], which is dependent on the physiological or pathophysiological status of the experimental animal. Recent studies have shown that $5-\mathrm{HT}_{1 \mathrm{~A}}$ has a protective effect against CIPN-related pain [16], and brain NTSR1 activation induced serotonergic neuronal activity and 5 -HT release $[11,17,18]$. Also, the antinociceptive effect of NTSR1 agonist administered to the rostral ventromedial medulla (RVM) on noxious thermal stimulus was inhibited by intrathecally administered non-selective serotonergic antagonist $[9,10]$. These results suggest that the antinociceptive effects of NTSRl agonist are mediated by interactions between NTSR1 and neurons that release 5-HT. Thus, the aim of the present study was to assess the role of NTSR1 in antinociception and to determine its association with the serotonergic pathway in a CIPN animal model at the spinal level.

\section{MATERIALS AND METHODS}

\section{Animals and the chemotherapy-induced neuropathic pain model}

Male Sprague-Dawley rats (weight 150-180 g) were purchased for this study. The animals were acclimated to the laboratory for 3-5 days before use. They were allowed free access to a standard rat diet and tap water in their home cage environment. The room was maintained at $24^{\circ} \mathrm{C}$ with a 12-hr/12-hr light/dark cycle. This study proposal was reviewed and approved by the Institutional Animal Care and Use Committee of Chonnam National University (IACUC$\mathrm{H}-2016-47)$. The rats were injected intraperitoneally with $2 \mathrm{mg} / \mathrm{kg}$ cisplatin for 4 days to induce the CIPN model, as described previously [19].

\section{Implantation of an intrathecal catheter for the injection route}

A length of $8.5 \mathrm{~cm}$ polyethylene-10 tube was inserted into the subarachnoid space under sevoflurane anesthesia through a small incision in the atlantooccipital membrane 5 days after the first cisplatin injection, as previously described [20]. The tip of the catheter was advanced to the lumbar enlargement. A recovery period of 9 days after implantation was provided before proceeding to the behavioral study. Rats with neurological damage were excluded.

\section{Drugs}

Cisplatin (Tocris Cookson Ltd., Bristol, Avon, UK) was freshly dissolved in $0.4 \%$ dimethyl sulfoxide (DMSO) to a final concentration of $2 \mathrm{mg} / \mathrm{mL}$. PD 149163 (Sigma-Aldrich, St. Louis, MO), a selective NTSR1 agonist, was dissolved in saline. SR 48692 (Tocris), a selective non-peptide NTSR1 antagonist, and dihydroergocristine (Tocris), a 5-HT receptor antagonist, were dissolved in $100 \%$ and $70 \%$ DMSO, respectively. The drugs were administered intrathecally in a $10-\mu \mathrm{L}$ solution using a microinjection syringe pump. The control group (vehicle) was given DMSO of equal volume and concentration. Intraperitoneal injections were performed with a $26 \mathrm{G}$ needle attached to a syringe.

\section{Assessment of mechanical allodynia}

Mechanical allodynia was tested after the rats recovered from intrathecal surgery. The rats were placed on a wire net cage individually to evaluate paw withdrawal thresholds. The rats were acclimated to the environment for 30 minutes before testing. Mechanical allodynia was measured with von Frey filaments (Stoelting, Wood Dale, IL) 
to the hind paw using the up-and-down method. A series of eight von Frey filaments (0.4, 0.6, 1, 2, 4, 6, 8, 15 g) was gently applied perpendicular to the plantar surface of the hind paw for 5 seconds after the filament was bent. Brisk withdrawal or paw flinching was regarded as a positive response. The absence of a positive response to the pressure of the $15 \mathrm{~g}$ von Frey filament was regarded as the cutoff value. Only rats with definite allodynia were tested (mechanical withdrawal threshold $<5 \mathrm{~g}$ after CIPN was induced). Test compounds were randomly administered. The same volume of vehicle (saline or DMSO) was administered to the control group animals. All rats were evaluated only once, and the investigator was blinded to the treatment status of all animals in the experimental tests.
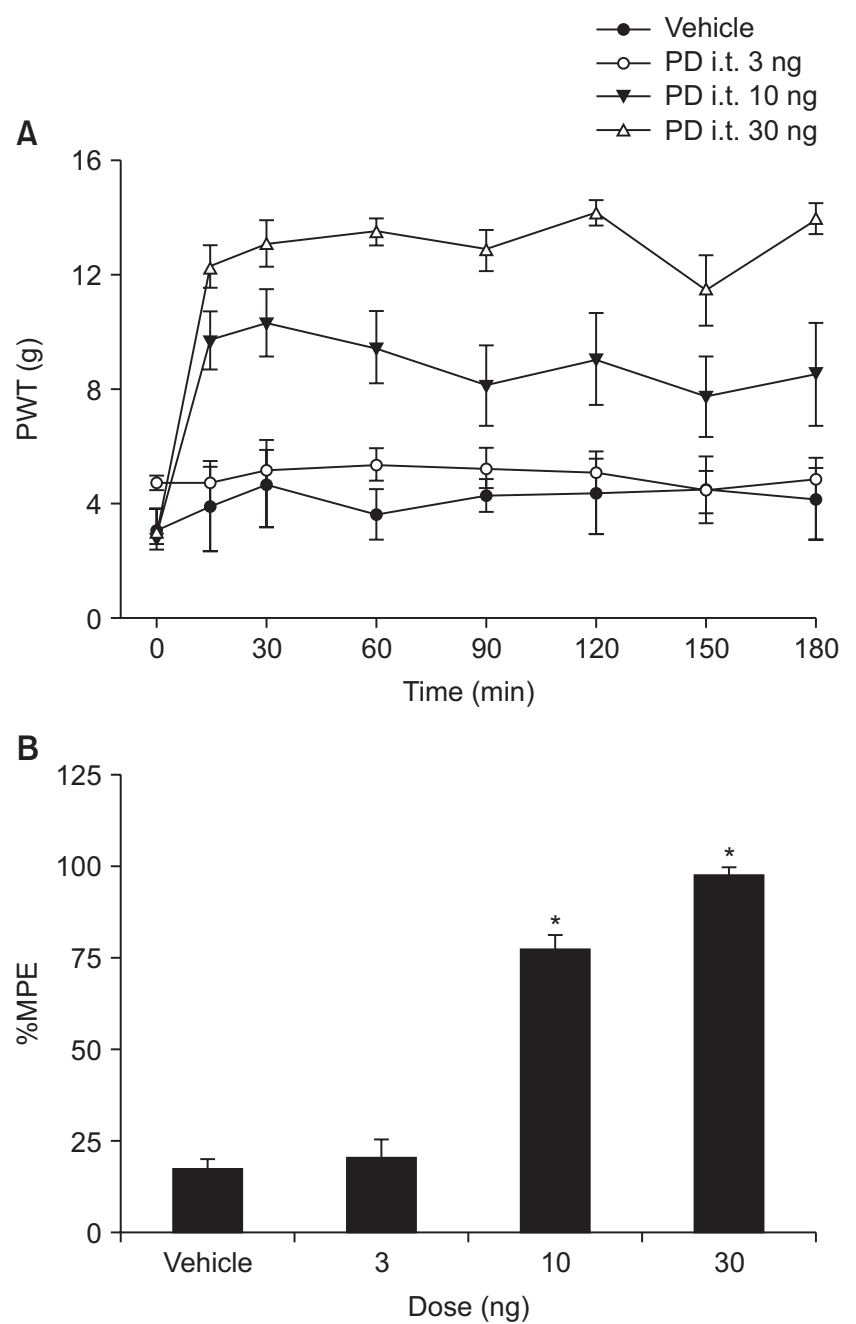

\section{General behavior}

Additional rats received the highest doses of the drugs to evaluate the behavioral changes after administration of each drug 1 hour after intrathecal or intraperitoneal delivery. Righting and place-stepping reflexes were observed to check motor functions, as described previously [21]. Pinna and corneal reflexes were judged as present or absent. Other abnormal behaviors, such as serpentine movement or tremors, were also evaluated.

\section{Assessment of serotonin concentration in the spinal cord}

The concentration of serotonin in the spinal cord was measured as described previously [22]. Tissue was obtained from the dorsal area of the lumbar (L4-5) spinal
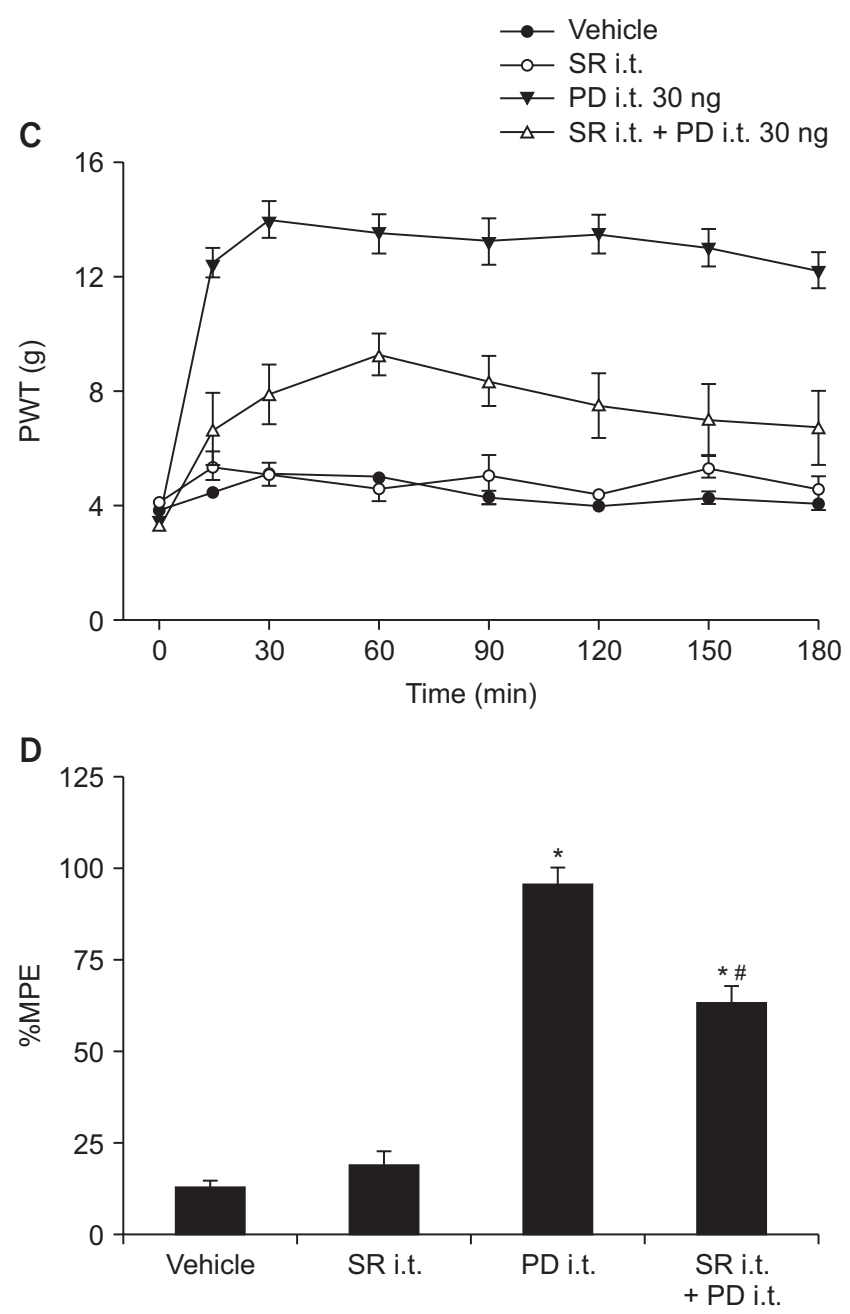

Fig. 1. Antinociceptive effect of intrathecal PD 149163 in chemotherapy-induced peripheral neuropathic rats. (A, B) The effect of intrathecal PD 149163 (PD: 0, 3, 10, and $30 \mathrm{ng}$ ) on the hind paw withdrawal threshold (PWT) to von Frey filaments. (C, D) The effect of intrathecal SR 48692 (SR: 0 or 100 $\mu \mathrm{g})$ on the antiallodynic effect caused by PD 149163 (30 ng). SR 48692 was delivered 10 minutes before PD 149163 administration. Each line or bar represents mean \pm standard error of 6-7 rats. MPE: maximum possible effect, i.t.: intrathecal administration. ${ }^{*} P<0.05$ compared to vehicle. ${ }^{\#} P<0.05$ compared to PD 149163 (i.t.)-treated group. 

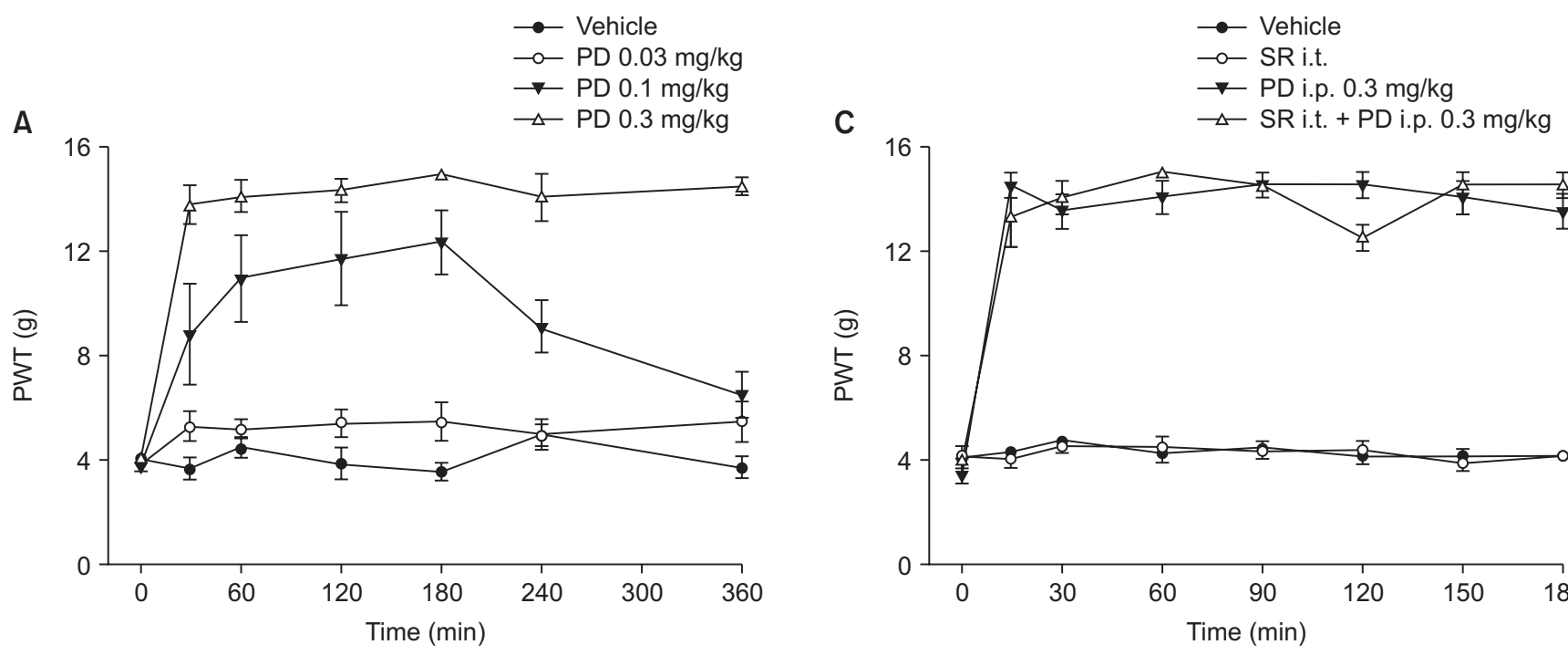

B
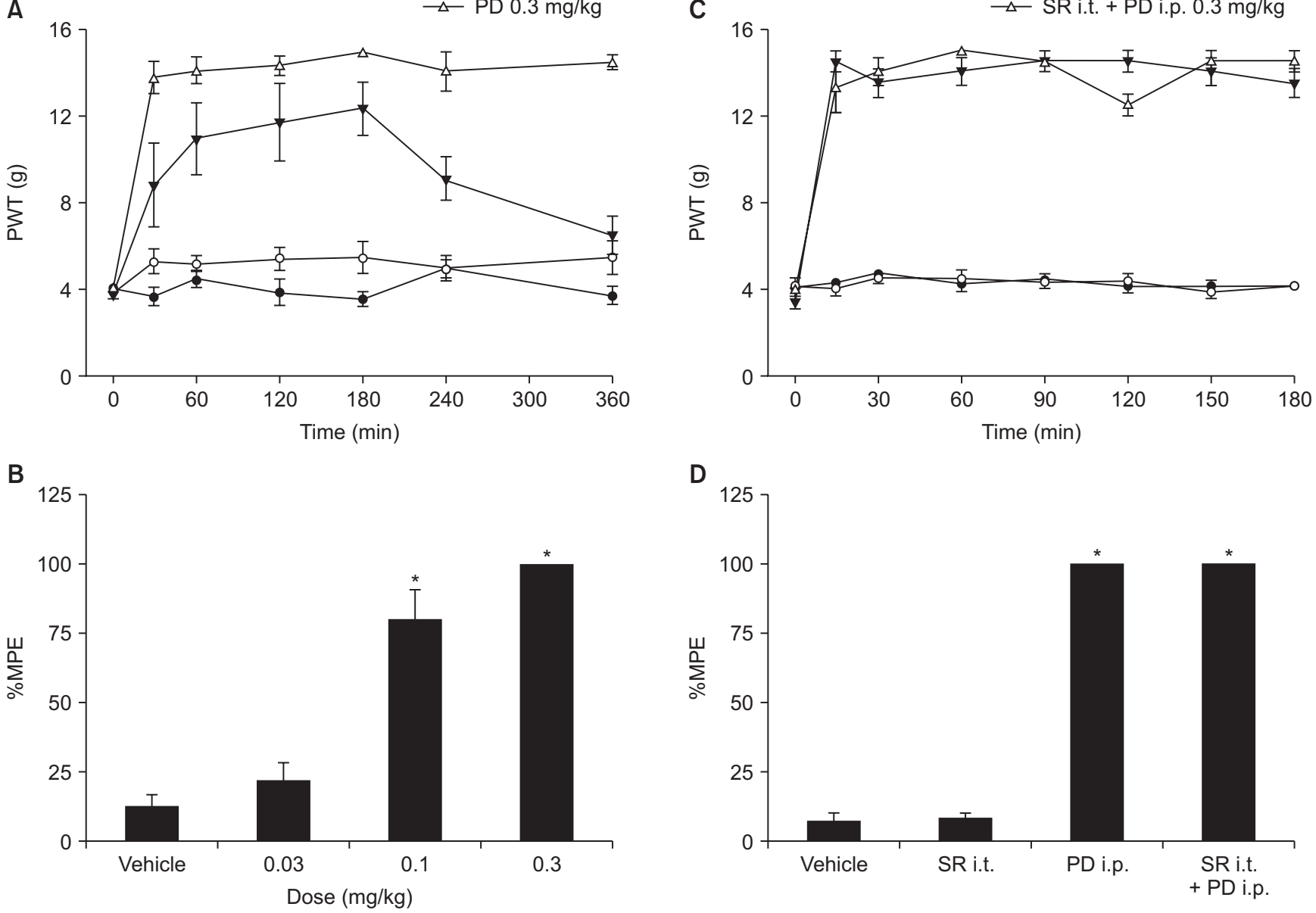

D

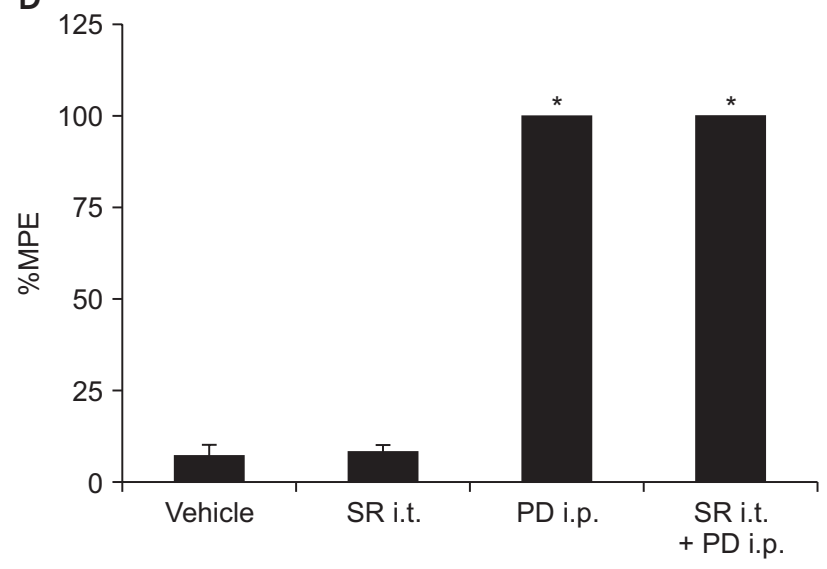

Fig. 2. Antinociceptive effect of intraperitoneal PD 149163 in chemotherapy-induced peripheral neuropathic rats. (A, B) The effect of intraperitoneal PD 149163 (PD: 0, 0.03, 0.1, and $0.3 \mathrm{mg} / \mathrm{kg}$ ) for the hind paw withdrawal threshold (PWT) to von Frey filaments. (C, D) The effect of intrathecal SR 48692 (SR: 0 or $100 \mu \mathrm{g}$ ) on the antiallodynic effect caused by intraperitoneal PD 149163 (0.3 mg/kg). SR 48692 was delivered 10 minutes before PD 149163 administration. Each line or bar represents mean \pm standard error from 6 rats. MPE: maximum possible effect, i.t.: intrathecal administration, i.p.: intraperitoneal administration. $* P<0.05$ compared to vehicle.

cord 14 days after the first injection of cisplatin. PD 149163 was administered intraperitoneally or intrathecally 2 hours before the harvest of spinal cord tissue. The serotonin concentration was detected using high-performance liquid chromatography and liquid chromatographytandem mass spectrometry systems supplied by Shimadzu (LC 20A System and API 4000 Q Trap; Tokyo, Japan). A 0.4$\mathrm{mL}$ aliquot of $0.5 \mathrm{M}$ formic acid was added to homogenate the tissue.

\section{Statistical analysis}

Data are expressed as mean \pm standard error. The doseresponse data are presented as the percentage of maximum possible effect (\%MPE): \%MPE = [(post-drug threshold - post-injury baseline threshold)/(cutoff threshold post-injury baseline threshold)] $\times 100$. The dose-response data and serotonin concentrations were analyzed by oneway analysis of variance with Tukey's post hoc test or the paired $t$-test. The statistical analysis was performed using IBM SPSS Statistics 22.0 software (IBM Corp., Armonk, NY). A $P$ value $<0.05$ was considered significant.

\section{RESULTS}

A total of 171 rats were used in this experiment. Placestepping and righting reflexes were observed at the highest dose used in this study, and normal, pinna and corneal reflexes were normally displayed as well. No overt abnormal behavioral changes were observed.

We initially investigated whether NTSR1 regulated the spinal nociceptive signal in the CIPN model. As shown in Fig. $1 \mathrm{~A}$ and $\mathbf{B}$, intrathecal administration of the selective 

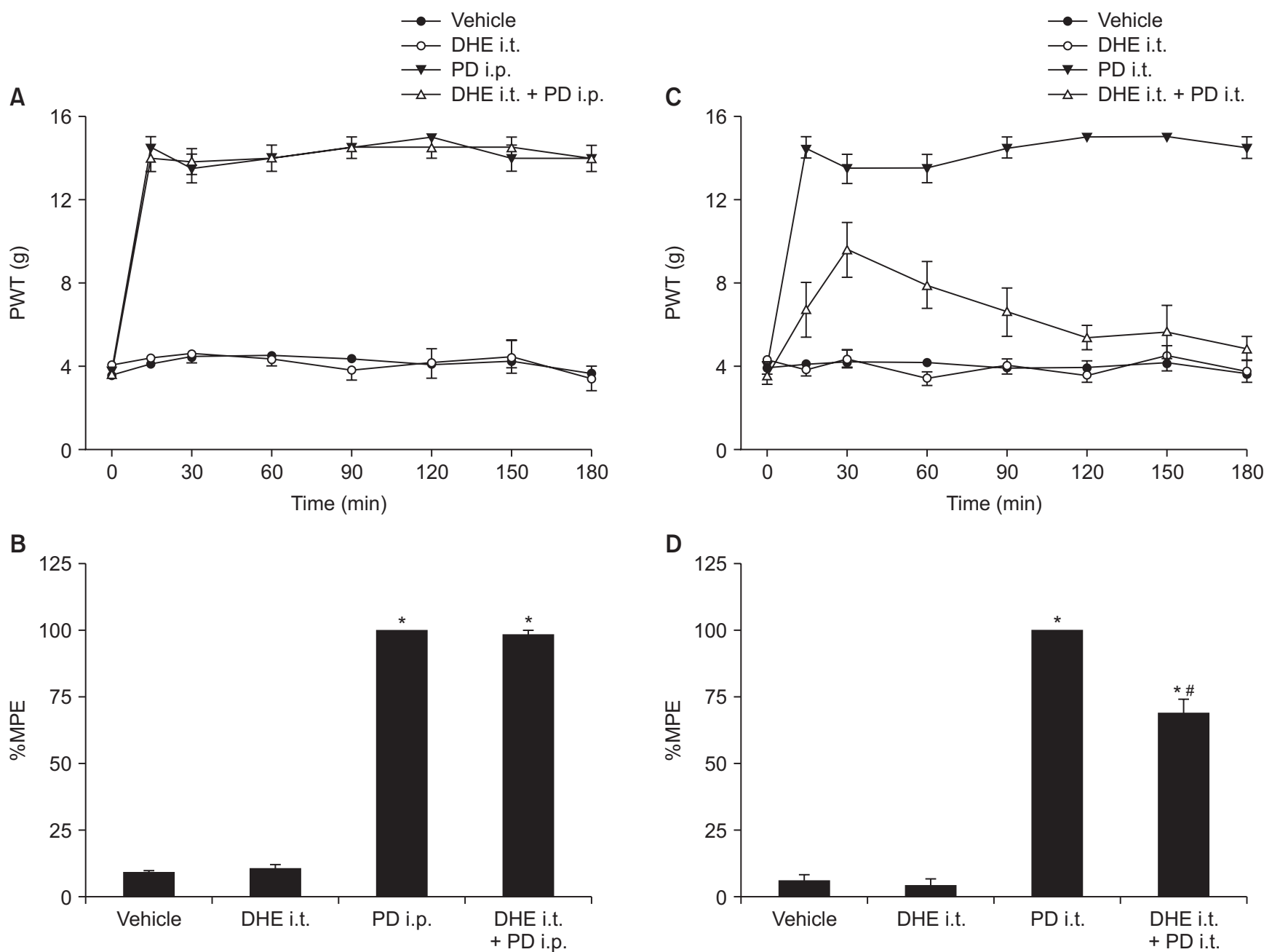

Fig. 3. Association between the serotonin (5-hydroxytryptamine) receptor and the antinociceptive effect of intraperitoneal or intrathecal administration of PD 149163. (A, B) The effect of intrathecal dihydroergocristine (DHE: 0 or $30 \mu \mathrm{g}$ ) on the antinociceptive effect of intraperitoneal PD 149163 (PD: $0.3 \mathrm{mg} /$ $\mathrm{kg})$. (C, D) The effect of intrathecal dihydroergocristine (30 $\mu \mathrm{g})$ on the antinociceptive effect of intrathecal PD 149163 (30 ng). Dihydroergocristine was delivered 10 minutes before PD 149163 administration. Each line or bar represents mean \pm standard error of 6-7 rats. PWT: paw withdrawal threshold, MPE: maximum possible effect, i.t.: intrathecal administration, i.p.: intraperitoneal administration. ${ }^{*} P<0.05$ compared to vehicle. ${ }^{\#} P<0.05$ compared to PD 149163 (i.t.)-treated group.

NTSR1 agonist PD 149163 dose-dependently and markedly improved mechanical allodynia induced by cisplatin. In addition, the selective NTSR1 antagonist SR 48692, administered intrathecally, inhibited the antinociceptive effects induced by PD 149163 (Fig. 1C, D). These results indicate that NTSR1 plays a critical role in regulating CIPN-related pain at the spinal cord level. Next, we administered PD 149163 into the peritoneal space, a common route of systemic drug administration. As shown in Fig. 2A and B, intraperitoneal administration of PD 149163 increased the paw withdrawal threshold in a dose-dependent manner. However, intrathecally administered SR 48692 did not inhibit the antinociceptive effects induced by intraperitoneally administered PD 149163 (Fig. 2C, D).

Previous studies have shown that the antinociceptive effects induced by PD 149163 injected directly into the brain are inhibited by intrathecal administration of a non-selective serotonergic antagonist [9]. Therefore, we examined whether the effect of systemic administration of the selective brain-penetrating NTSRl agonist PD 149163 in the CIPN model could be blocked by intrathecal administration of dihydroergocristine, a non-selective serotonergic antagonist. As shown in Fig. 3A and B, the antinociceptive effects of intraperitoneally administered PD 149163 were not inhibited by intrathecal administration of dihydroergocristine. However, when PD 149163 was administered intrathecally, the beneficial effect of PD 149163 on mechanical allodynia was diminished by intrathecally administered dihydroergocristine (Fig. 3C, D). Serotonin concentration at the spinal cord level decreased in CIPNinduced rats compared to the controls, but intrathecal or intraperitoneal administration of PD 149163 did not affect 


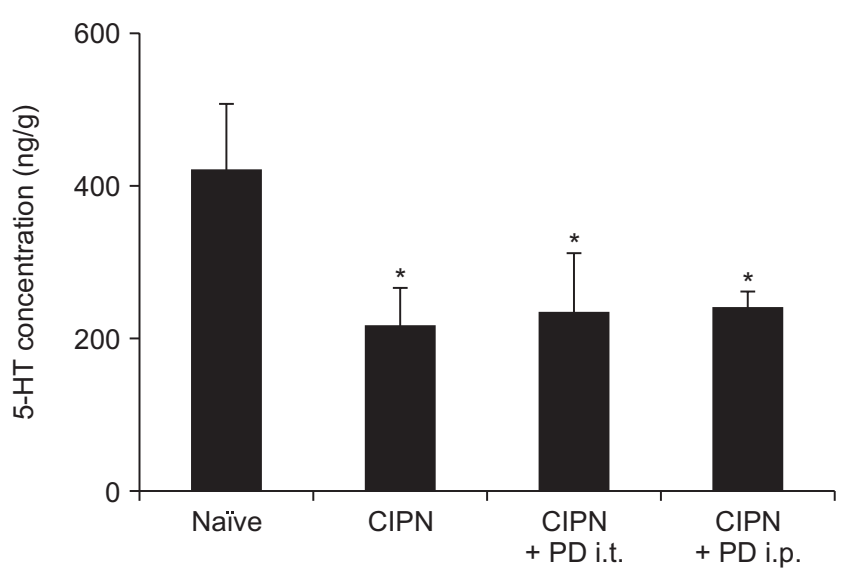

Fig. 4. The levels of serotonin (5-hydroxytryptamine, 5-HT) in the spinal cord of chemotherapy-induced peripheral neuropathic (CIPN) rats. The rats were divided into four groups: Naïve group, CIPN group, CIPN group treated with intrathecal PD 149163 (PD), CIPN group treated with intraperitoneal PD 149163. Each bar represents mean \pm standard error of 6 rats. i.t.: intrathecal administration, i.p.: intraperitoneal administration. $* P<0.05$ compared to naïve rats.

the observed decrease (Fig. 4).

\section{DISCUSSION}

The present study demonstrated potent anti-allodynic effects of intrathecal and intraperitoneal NTSRl agonist in CIPN, and provided evidence that the NTSR1 receptor plays significant roles in spinal- and supraspinal-level pain processing. The anti-allodynic effect of intrathecal PD 149163 was reduced by intrathecal pretreatment with the NTSR1 antagonist SR 48692, which parallels the analgesic effect shown in previous studies using the formalin test and sciatic nerve constriction model [12,14]. In addition, these previous studies showed that NTSR1 is distributed in the dorsal root ganglia and the superficial layers of the dorsal horn at the spinal cord [12]. We also examined the effect of the intrathecal NTSR2 agonist levocabastine in the CIPN model, but it did not affect mechanical allodynia intensity (data not shown). Moreover, a selective agonist of NSTR3 has not been developed, so it could not be added to this experiment. Therefore, these results indicate that NTSR1, not NTSR2, is involved in the regulation of the chemotherapy-evoked nociceptive transmission in the spinal cord. However, previous studies showed that intrathecal NTSR2 agonists, including JMV431, levocabastine, or $\beta$-lactotensin, produced analgesic effects in sciatic nerve injury- and formalin-induced pain models [23,24]. Although such discrepancy on the effect of NTSR2 agonist could not be exactly understood, it may be due to the kinds of drug and nociception. Thus, further research is necessary in future.
NTS has little analgesic effect when administered peripherally because it is readily degraded by peptidases in the periphery, and does not easily pass the bloodbrain barrier [25,26]. However, PD 149163 is a stable and brain penetrating NTSR1 agonist [27]. As shown in our experimental data, intraperitoneal administration of PD 149163 effectively abolished mechanical allodynia. However, intrathecal SR 48692 had no influence on the effect of intraperitoneal PD 149163. These results suggest that intraperitoneal PD 149163 mainly acts on brain NTSR1 and its supraspinal analgesic action was sufficient to suppress neuropathic pain in the CIPN model. NTS is widely distributed in the CNS, and acts as a neurotransmitter in the brain. For example, systemic administration of NTSR1 agonists regulate various CNS disorders, including schizophrenia [28]. In addition, direct administration of NTS into specific brain regions (periaqueductal gray region and RVM) exerted strong analgesic effects $[29,30]$.

Previous studies have demonstrated NTSR1 regulation of serotonergic neuronal activity and 5-HT release [11,17]. NTS increases 5-HT release in brain frontal cortex slices, which was inhibited by NTSR1 antagonists [17]. Local injection of NTS in the prefrontal cortex dose-dependently increased 5-HT release in freely moving rats, which was also inhibited by SR 48692 [11]. It is possible that supraspinal NTSR1 is involved in descending serotonergic pain control. Indeed, another study found that direct injection of NTS or PD 149163 in the RVM produced an antinociceptive effect in the thermal tail flick test. Intrathecal administration of a non-selective serotonergic antagonist blocked this effect, which suggests that increased 5-HT release from spinally projecting serotonergic neurons in the RVM is responsible for the antinociceptive effect of RVM NTSR1 agonists [9].

However, in our study using the CIPN model, the antiallodynic effect of intraperitoneal PD 149163 was not affected by pretreatment with intrathecal dihydroergocristine, a non-selective serotonergic antagonist. This could stem from different pain modalities: acute thermal nociception in naïve animals versus mechanical allodynia in the neuropathic CIPN model. Compared with naïve animals, the CIPN animals showed a significant decrease in spinal cord 5-HT content. Thus, activation of NTSR1 in the brain did not increase 5-HT in CIPN rats to the level of naïve animals. In addition, intraperitoneal PD $149163 \mathrm{had}$ no effect on spinal cord serotonin levels in our CIPN model. Together, these results provide evidence for pathologic changes in the serotonergic descending inhibitory pathway in CIPN. Another possibility is that the intraperitoneal NTSR1 agonist may activate the noradrenergic descending inhibitory pathway. Support for this possibility stems from recent studies demonstrating that local injection of 
NTSRl agonist to the brain partially inhibited the thermal antinociceptive effect via the intrathecal $\alpha 2$-adrenoceptor antagonist [10].

In contrast to the anti-allodynic effect of the intraperitoneal NTSRl agonist, which was independent of spinal level serotonergic action, intrathecal administration of the NTSR1 agonist diminished the effect of the intrathecal nonspecific 5-HT antagonist. It is possible that the intrathecal 5-HT antagonist further suppressed the spinal serotonergic modulating system responsible for reduction of spinal 5-HT content. These data suggest that the basal activity of the serotonergic receptor is required for the NTSR1 agonist to exhibit a maximal antinociceptive effect in the spinal cord. However, NTSR1 agonist may not directly affect the synthesis of serotonin.

Collectively, our study demonstrates that PD 149163 has a strong anti-allodynic effect in a rodent model of CIPN at the supraspinal as well as the spinal level and provides new therapeutic targets for effective CIPN analgesics. In particular, the effect of PD 149163 may be indirectly related to the serotonergic pathway.

\section{CONFLICT OF INTEREST}

No potential conflict of interest relevant to this article was reported.

\section{FUNDING}

This study was supported by a grant (BCRI16022-1) of Chonnam National University Hospital Biomedical Research Institute.

\section{ORCID}

Mei Yin, https://orcid.org/0000-0002-0564-8434

Yeo-Ok Kim, https://orcid.org/0000-0002-1748-8079

Jeong-Il Choi, https://orcid.org/0000-0002-3571-2599

Seongtae Jeong, https://orcid.org/0000-0002-6245-9779

Si-Ho Yang, https://orcid.org/0000-0002-4193-4438

Hong-Beom Bae, https://orcid.org/0000-0002-0358-6807

Myung-Ha Yoon, https://orcid.org/0000-0002-9962-0255

\section{REFERENCES}

1. Flatters SJL, Dougherty PM, Colvin LA. Clinical and preclinical perspectives on Chemotherapy-Induced Peripheral Neuropathy (CIPN): a narrative review. Br J Anaesth 2017; 119:
737-49.

2. Addington J, Freimer M. Chemotherapy-induced peripheral neuropathy: an update on the current understanding. F1000Res 2016; 5: F1000 Faculty Rev-1466.

3. Fukuda Y, Li Y, Segal RA. A mechanistic understanding of axon degeneration in chemotherapy-induced peripheral neuropathy. Front Neurosci 2017; 11: 481.

4. Bhandare AM, Kshirsagar AD, Vyawahare NS, Hadambar AA, Thorve VS. Potential analgesic, anti-inflammatory and antioxidant activities of hydroalcoholic extract of Areca catechu L. nut. Food Chem Toxicol 2010; 48: 3412-7.

5. Paice JA. Clinical challenges: chemotherapy-induced peripheral neuropathy. Semin Oncol Nurs 2009; 25(2 Suppl 1): S8-19.

6. Hile ES, Fitzgerald GK, Studenski SA. Persistent mobility disability after neurotoxic chemotherapy. Phys Ther 2010; 90: 1649-57.

7. Carraway R, Leeman SE. The isolation of a new hypotensive peptide, neurotensin, from bovine hypothalami. J Biol Chem 1973; 248: 6854-61.

8. Jennes L, Stumpf WE, Kalivas PW. Neurotensin: topographical distribution in rat brain by immunohistochemistry. J Comp Neurol 1982; 210: 211-24.

9. Buhler AV, Choi J, Proudfit HK, Gebhart GF. Neurotensin activation of the NTR1 on spinally-projecting serotonergic neurons in the rostral ventromedial medulla is antinociceptive. Pain 2005; 114: 285-94.

10. Buhler AV, Proudfit HK, Gebhart GF. Neurotensin-produced antinociception in the rostral ventromedial medulla is partially mediated by spinal cord norepinephrine. Pain 2008; 135: 280-90.

11. Petkova-Kirova P, Rakovska A, Zaekova G, Ballini C, Corte LD, Radomirov R, et al. Stimulation by neurotensin of dopamine and 5-hydroxytryptamine (5-HT) release from rat prefrontal cortex: possible role of NTR1 receptors in neuropsychiatric disorders. Neurochem Int 2008; 53: 355-61.

12. Roussy G, Dansereau MA, Doré-Savard L, Belleville K, Beaudet N, Richelson E, et al. Spinal NTS1 receptors regulate nociceptive signaling in a rat formalin tonic pain model. J Neurochem 2008; 105: 1100-14.

13. Smith KE, Boules M, Williams K, Richelson E. NTS1 and NTS2 mediate analgesia following neurotensin analog treatment in a mouse model for visceral pain. Behav Brain Res 2012; 232: 93-7.

14. Guillemette A, Dansereau MA, Beaudet N, Richelson E, Sarret $\mathrm{P}$. Intrathecal administration of NTS1 agonists reverses nociceptive behaviors in a rat model of neuropathic pain. Eur J Pain 2012; 16: 473-84.

15. Bardin L. The complex role of serotonin and 5-HT receptors in chronic pain. Behav Pharmacol 2011; 22: 390-404.

16. Ward SJ, McAllister SD, Kawamura R, Murase R, Neelakan$\tan \mathrm{H}$, Walker EA. Cannabidiol inhibits paclitaxel-induced 
neuropathic pain through 5-HT(1A) receptors without diminishing nervous system function or chemotherapy efficacy. Br J Pharmacol 2014; 171: 636-45.

17. Héaulme M, Leyris R, Soubrié P, Le Fur G. Stimulation by neurotensin of $(3 \mathrm{H}) 5$-hydroxytryptamine (5HT) release from rat frontal cortex slices. Neuropeptides 1998; 32: 465-71.

18. Jolas T, Aghajanian GK. Neurotensin excitation of serotonergic neurons in the dorsal raphe nucleus of the rat in vitro. Eur J Neurosci 1996; 8: 153-61.

19. Lin H, Heo BH, Yoon MH. A new rat model of cisplatininduced neuropathic pain. Korean J Pain 2015; 28: 236-43.

20. Yaksh TL, Rudy TA. Chronic catheterization of the spinal subarachnoid space. Physiol Behav 1976; 17: 1031-6.

21. Han YK, Lee SH, Jeong HJ, Kim MS, Yoon MH, Kim WM. Analgesic effects of intrathecal curcumin in the rat formalin test. Korean J Pain 2012; 25: 1-6.

22. Kim YO, Song JA, Kim WM, Yoon MH. Antiallodynic effect of intrathecal Korean Red Ginseng in cisplatin-induced neuropathic pain rats. Pharmacology 2020; 105: 173-80.

23. Tétreault P, Beaudet N, Perron A, Belleville K, René A, Cavelier $F$, et al. Spinal NTS2 receptor activation reverses signs of neuropathic pain. FASEB J 2013; 27: 3741-52.

24. Roussy G, Dansereau MA, Baudisson S, Ezzoubaa F, Bel- leville K, Beaudet N, et al. Evidence for a role of NTS2 receptors in the modulation of tonic pain sensitivity. Mol Pain 2009; 5: 38.

25. Boules M, McMahon B, Warrington L, Stewart J, Jackson J, Fauq A, et al. Neurotensin analog selective for hypothermia over antinociception and exhibiting atypical neurolepticlike properties. Brain Res 2001; 919: 1-11.

26. Demeule M, Beaudet N, Régina A, Besserer-Offroy É, Murza $A$, Tétreault $P$, et al. Conjugation of a brain-penetrant peptide with neurotensin provides antinociceptive properties. J Clin Invest 2014; 124: 1199-213.

27. Banks WA, Wustrow DJ, Cody WL, Davis MD, Kastin AJ. Permeability of the blood-brain barrier to the neurotensin8-13 analog NT1. Brain Res 1995; 695: 59-63.

28. Hillhouse TM, Prus AJ. Effects of the neurotensin $\mathrm{NTS}_{1}$ receptor agonist PD 149163 on visual signal detection in rats. Eur J Pharmacol 2013; 721: 201-7.

29. Clineschmidt BV, Martin GE, Veber DF. Antinocisponsive effects of neurotensin and neurotensin-related peptides. Ann NY Acad Sci 1982; 400: 283-306.

30. Fang FG, Moreau JL, Fields HL. Dose-dependent antinociceptive action of neurotensin microinjected into the rostroventromedial medulla of the rat. Brain Res 1987; 420: 171-4. 